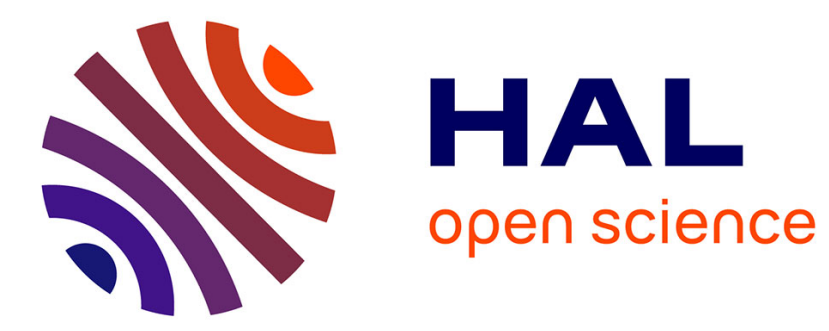

\title{
Do it yourself: make your own thermoacoustic engine with steel wool or rice
}

Olivier Come, Gaelle Poignand, Guillaume Penelet

\section{To cite this version:}

Olivier Come, Gaelle Poignand, Guillaume Penelet. Do it yourself: make your own thermoacoustic engine with steel wool or rice. European Journal of Physics, 2017, 38 (1), 10.1088/01430807/38/1/015101 . hal-01397360

\section{HAL Id: hal-01397360 \\ https://hal.science/hal-01397360}

Submitted on 15 Nov 2016

HAL is a multi-disciplinary open access archive for the deposit and dissemination of scientific research documents, whether they are published or not. The documents may come from teaching and research institutions in France or abroad, or from public or private research centers.
L'archive ouverte pluridisciplinaire HAL, est destinée au dépôt et à la diffusion de documents scientifiques de niveau recherche, publiés ou non, émanant des établissements d'enseignement et de recherche français ou étrangers, des laboratoires publics ou privés. 


\title{
Do It Yourself: make your own thermoacoustic engine with steel wool or rice.
}

\author{
Olivier C, Poignand G and Pénelet G \\ Laboratoire d'Acoustique de l'Université du Maine UMR CNRS-6613, Avenue \\ Olivier Messiaen, 72085 Le Mans Cedex 9, France \\ E-mail: come.olivier@univ-lemans.fr
}

\begin{abstract}
The construction of two demonstrators of thermoacoustic engines is proposed in this paper, using common material from hardware stores. A brief discussion about the thermoacoustic effect highlights the main parameters controlling the generation of self-sustained acoustic oscillations from heat. The construction of the two tabletop demonstrators is then described in detail, so that they can be reproduced with minimum equipment (rice, steel wool, and other hardware easy to find in hardware stores). Experimental results are also presented for both engines and a discussion about their use for teaching purpose is also provided.
\end{abstract}

PACS numbers: 43.35.Ud

Keywords: Thermoacoustics, Self-sustained oscillator, DIY

The final publication is available at Springer via http://dx.doi.org/10.1088/ $0143-0807 / 38 / 1 / 015101$

\section{Introduction}

There is a large number of problems in acoustics in which considering the propagation of sound as an adiabatic process is a good assumption. It is usual to characterise the acoustic process by means of pressure and velocity fluctuations, but a gas parcel submitted to an acoustic wave also experiences temperature (and density) fluctuations. The temperature of a gas parcel compressed adiabatically rises (as can be experienced when operating a bicycle hand pump), while adiabatic expansion leads to a temperature decrease (think of the cold gas coming out of a spray can). Still considering an adiabatic process, the temperature fluctuations $\tau(t)$ of a gas parcel can be easily calculated from its pressure fluctuations $p(t)$, using the relation [1, 2] $p=\rho_{0} C_{p} \tau$, where $\rho_{0}$ and $C_{p}$ respectively stand for the mean (non-oscillating) density and for the isobaric heat capacity of the fluid. Having in mind an order of magnitude of the amplitude of these fluctuations is important: if one considers for instance a plane wave propagating through air under standard conditions (static pressure $P_{0}=10^{5} \mathrm{~Pa}$, temperature $T_{0}=300 \mathrm{~K}$ ) with frequency $f=200 \mathrm{~Hz}$, and if the associated pressure level is fixed to the threshold of pain for human hearing $20 \log _{10}\left(p_{r m s} / p_{0}\right)=120 \mathrm{~dB}$ SPL where $p_{0}=2 \times 10^{-5} \mathrm{~Pa}$ stands for the pressure at the human hearing threshold, then the peak amplitude of pressure fluctuations is equal to $p_{p}=20 \sqrt{2} \mathrm{~Pa}$, the peak amplitude of gas displacement equals $\xi_{p}=p_{p} /\left(2 \pi \rho_{0} c_{0} f\right) \approx 50 \mu \mathrm{m}$ where $c_{0}$ stands 
for the adiabatic sound speed, while the peak amplitude of temperature fluctuation, equals $\tau_{p} \approx 23 \mathrm{mK}$. From these values, one can conclude that temperature (as well as pressure) fluctuations are very small even at large acoustic levels, but it is also noticeable that the temperature gradient experienced by a gas parcel during half an acoustic cycle, namely $\tau_{p} / \xi_{p} \approx 460 \mathrm{~K} / \mathrm{m}$, is not small.

However, the assumption of an adiabatic process fails as soon as the propagation of an acoustic wave through a porous medium is considered. In such situation, the thermal coupling between the solid frame and the oscillating fluid leads to sound attenuation due to irreversible heat exchanges between the fluid and the solid, taking place within the so-called thermal boundary layers. Actually, and although the heat conductivity of a gas is generally very small (typically $26 \mathrm{~mW} / \mathrm{m} / \mathrm{K}$ for air), the losses due to heat diffusion have the same order of magnitude than the viscous losses in porous materials. The mechanism by which acoustic energy is dissipated is a relaxation process, which itself is due to the existence of a phase shift between pressure and temperature fluctuations in the vicinity of the solid walls. Due to this phase shift, the small amount of heat that a gas parcel gives to the solid during its phase of greatest condensation is larger than the amount of heat given by the solid to the gas parcel during its phase of greatest rarefaction.

There is nonetheless a way to transform attenuation into amplification of sound if one finds a mean to provide heat to the gas parcel during its phase of greatest condensation, and (partly) take it away during its phase of greatest rarefaction. This amplification process was identified more than one century ago by Lord Rayleigh in his famous textbook [3] when trying to explain qualitatively the operation of devices giving rise to sound generation from heat, such as the Sondhauss tube 4] and the Rijke tube [5. More generally, the phase relationship between pressure oscillations and the rate of unsteady heat released to the gas is a key control parameter (now referred to as the Rayleigh criterion) which governs the generation of sound from heat. This Rayleigh criterion prevails for the description of combustion instabilities in gas turbines and rocket engines [6], as it also prevails for thermoacoustic engines which are within the scope of this article.

A sketch of a simple thermoacoustic engine is provided in figure 1. The basic principle of such a device involves the application of a steep temperature gradient along a porous medium (the so-called stack) inserted inside an acoustic resonator: if the temperature has the appropriate sign and amplitude (typically larger than $\tau_{p} / \xi_{p}$ ), then the thermal coupling between the fluid and the gas may give rise to the reversal of natural heat exchange processes, or in other words (and accordingly with the Rayleigh criterion) to the promotion of acoustic oscillations from the provision of heat during the phase of greatest condensation of a gas parcel. This will lead to the onset of selfsustained acoustic oscillations at the frequency of the most unstable acoustic mode of the device.

Though nowadays the thermoacoustic effect (more precisely the one involved in thermoacoustic engines) is studied by an international scientific community, it has long been considered as a curiosity. It was probably first observed by glass-blowers that their cold blowing cane would sometimes emit a low pitch continuous sound as a molten glass gob was mounted on it. The oldest evidence of a thermoacoustic engine strictly speaking comes from 16th century Japan, with the diary of a Buddhist monk referring to an object called "Kibitsunokama", which translates as "Kettle of Kibitsu". A collection of tales published in 1776 [7] gives a better description of it: this instrument, that is still used today during ceremonies in the Shintoist temple 
(a)

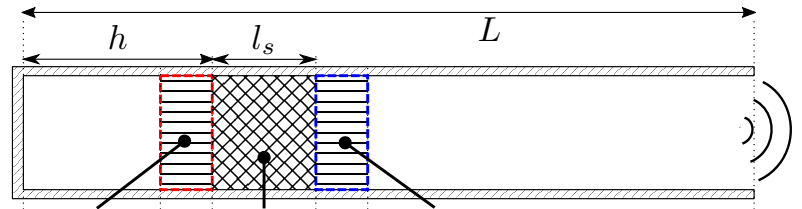

hot heat exchanger stack ambiant heat exchanger

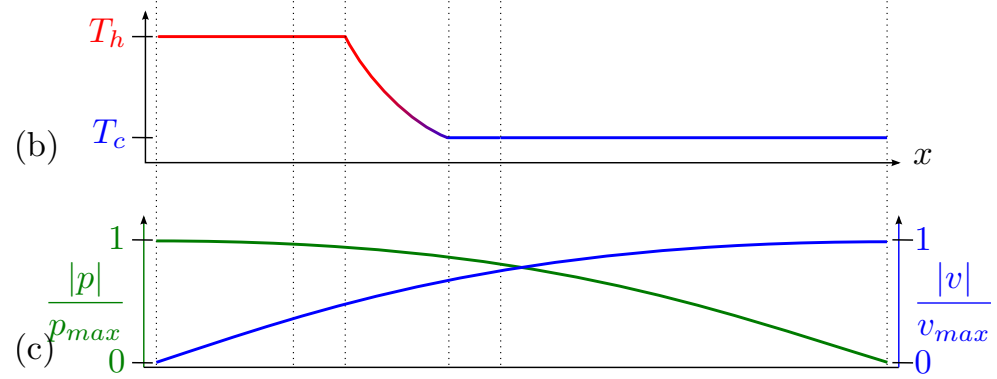

Figure 1. (a) Schematic representation of quarter-wave length thermoacoustic engine with main dimensions. (b) (Arbitrary) temperature distribution in the fluid, imposed by a couple of heat exchangers, varying between $T_{c}$ and $T_{h}$. (c) Schematic representation of the amplitude distribution of acoustic pressure $p$ (green, maximum at closed end and null at open end) and particular velocity $v$ (blue, null at closed end and maximum at open end), normalised by their maximum value.

of Kibitsu, consists of a barrel mounted on an iron bowl containing water. A mesh screen in the barrel is loaded with rice. Put on a fire, the Kibitsunokama will emit a sound much like the bellowing of an ox [7]. The objective of this paper is neither to provide a detailed description of the thermoacoustic effect (which can be found elsewhere $8,9,10$ ) nor to present new research results on the topic, but it is rather to explain how to build two simple versions of the devices mentioned above with minimum equipment.

Several tabletop build-ups of thermoacoustic engines (and refrigerators) have been proposed in the literature [11, 12, 13, 14, 15, but they usually require either materials that are not commonly available (such as square pore ceramics used in catalytic exhaust systems, or machined parts of complex geometry) or minimum scientific hardware (e.g. a DC power supply). The two devices described in this paper are portable (they are compact and do not necessary require an electrical input) and very easy to build using widely available materials. We believe that these two devices could be used easily for teaching purpose in order to illustrate basic concepts of acoustics, thermodynamics, or self-sustained oscillations. Section 2 presents the most important design guidelines for building a simple thermoacoustic oscillator. The construction of a test tube thermoacoustic engine (a modern version of the Sondhauss tube) is presented in section 3, and a Kibitsunokama rice-based thermoacoustic engine is described in section 4. Finally, a conclusion is provided in section 5. where the possible use of these two devices for teaching purposes is highlighted.

\section{Basic principles and design guidelines}

Two operating principles are to be distinguished for the thermoacoustic effect, according to the conversion process. In both cases, the review paper 9 is a good 
starting point for more complete information.

The first case consists in sustaining a large amplitude acoustic wave through a porous material initially at uniform temperature. The porous material is usually called a stack, because first realisations were made of a pile of thin plates (but any open cell porous media could be used instead). The properly phased displacement and variations of pressure of a small volume of gas allows for it to give away a bit of its heat to the surrounding colder walls when the gas is compressed and "hot", and further take away a bit of heat from the slightly warmer walls when the gas parcel is dilated and cold. By a cumulative effect, heat is advected from an extremity of the stack to the other and a temperature gradient gradually emerges. This use of an acoustic work to produce a thermal gradient is the definition of a thermoacoustic heat-pump or refrigerator, whether the hot or cold side of the system is used. These systems will not be treated further here. Instructions to build a tabletop demonstrator of thermoacoustic refrigerator may be found in reference [16].

The second case, the one we are interested in, is the reciprocate effect. Consider a gas parcel oscillating in a porous material subject to a temperature gradient $\Delta T / l_{s}$ along the stack of length $l_{s}$. For a sufficiently large temperature difference $\Delta T$ along the stack, and with the proper phase-shift between its displacement and the pressure oscillation, this parcel receives a small amount of heat from the stack when it is compressed and hot, and further gives away a bit of heat to the colder stack when it is dilated and cold. The gas oscillation is thus amplified by the presence of the temperature gradient in the stack. If this thermoacoustic amplification is large enough to compensate all losses in the system, self-sustained oscillations are generated at the frequency of the most unstable mode of the acoustic network. This system is then called a thermoacoustic prime-mover or engine.

At the threshold of the autonomous oscillations, the behaviour of thermoacoustic engines with a known geometry (in particular that of the pores of the stack) can be described thanks to the linear theory of thermoacoustics 8,9 . This theory however fails to predict accurately the behaviour once the self-sustained instability is initiated. Indeed, the saturation of the oscillation in the singing engine is piloted by different nonlinear effects due to the high acoustic amplitudes (amongst them: acoustically enhanced heat conduction, acoustically induced streaming, wave steepening or vortex generation at the ends of the stack). Therefore, the development of an analytical model able to predict accurately the operation of a thermoacoustic engine is a hard task [17], even for the simple demonstrators presented hereafter.

Nevertheless, some general principles for the configurations presented here can be considered. In this paper, we will limit ourselves to the simple configuration of a stack placed into a resonator open at one end and closed at the other. A schematic view of such a quarter-wavelength thermoacoustic engine is given in figure 1, showing the main elements and dimensions for its characterisation. In this configuration, the working frequency $f$ of the engine is given by the length of the open/close resonator, and is approximated as $f \simeq c_{0} / 4 L$, where $c_{0} \simeq 346 \mathrm{~m} \mathrm{~s}^{-1}$ is the speed of sound in air at room temperature.

The stack is where the thermoacoustic conversion takes place. It must allow the passage of the acoustic wave and therefore have open pores with a low resistance to fluid flow. The pore size, which is evaluated by the average hydraulic radius $r_{h}$ (ratio of the cross sectional area to the wet perimeter), must be such that the heat exchanges between the gas subject to a standing wave and the solid walls are quasi-adiabatic. This condition is fulfilled for a pore size of a few times the thermal 


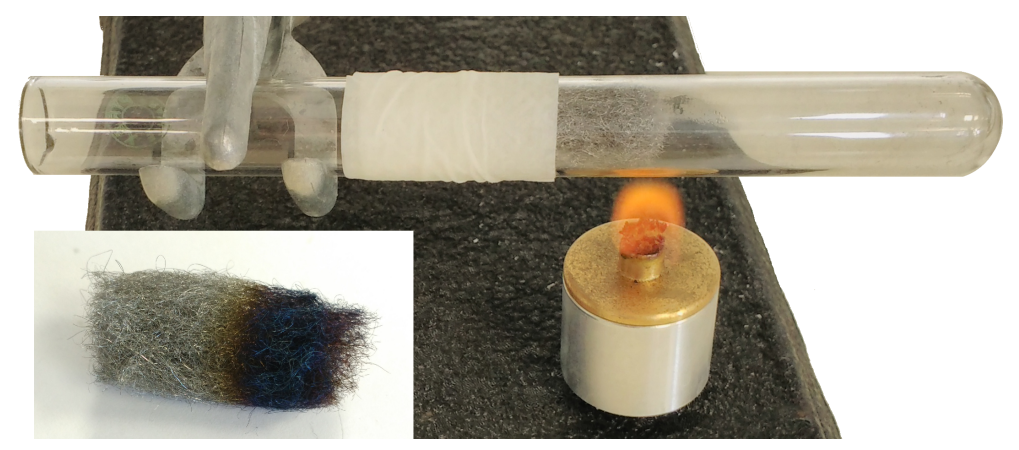

Figure 2. Thermoacoustic engine built from a test tube and a small piece of fine or medium steel wool (enlarged at bottom left). A strip of wet paper towel is wrapped around the cold extremity of the stack to maintain its temperature. The heat source is a small spirit lamp, or a lighter. The color of the steel is a good indicator of the temperature reached at the hot extremity of the stack: straw yellow indicates a temperature of $\sim 210^{\circ} \mathrm{C}$, brown indicates $\sim 260^{\circ} \mathrm{C}$ and deep blue $\sim 310^{\circ} \mathrm{C}[18$.

boundary layer thickness $r_{h} \sim 2.5 \delta_{\kappa}$, the latter being frequency dependent such that $\delta_{\kappa} \sim 2.6 \times 10^{-3} / \sqrt{f}$ for air at ambient pressure.

For the thermoacoustic amplification to take place, the temperature gradient has to be imposed appropriately. For the case of a quarter wavelength thermoacoustic prime-mover considered in this paper, the temperature gradient must be oriented accordingly with the axial gradient of pressure amplitude of the acoustic mode (otherwise damping would occur instead of amplification). Since thermoacoustic amplification depends on both the relative amplitude of pressure and velocity fluctuations, there exists an optimum position of the stack along the waveguide to minimise the threshold or to maximise the efficiency of power conversion. In the examples described below, the optimum position of the stack is around one quarter to one third of the length of the resonator, $h_{\text {opt }} \approx L / 4$ or $L / 3$, starting from the rigid plug (see figure 1).

\section{Test tube thermoacoustic engine}

Following the principles given above, one of the simplest thermoacoustic engine can be built with very few materials, namely steel wool, a test tube and a burner.

\subsection{Making it}

The configuration pictured on figure 2 uses a $140 \mathrm{~mm}$ long pyrex test tube of $14 \mathrm{~mm}$ in diameter. The stack is a $20 \times 40 \mathrm{~mm}^{2}$ fine steel wool rectangle, cut in a common unrolled steel wool pad. The rectangle is rolled along its width and inserted to three quarters of the length of the tube (i.e. at $4.5 \mathrm{~cm}$ of the end). The tube is heated below the end of the stack on the closed side of the tube with a flame, either a lighter or a small spirit lamp. The other side of the stack is maintained at ambient temperature by a soaked paper towel strip rolled around the pipe. A short video clip, showing the fabrication and a run of the engine, has been made available at https://youtu.be/owbjLWrC86g. Built with a $14 \mathrm{~cm}$ tube, this engine sings at a frequency close to $f \simeq c_{0} / 4 L=630 \mathrm{~Hz}$. The average pore size for which the onset 


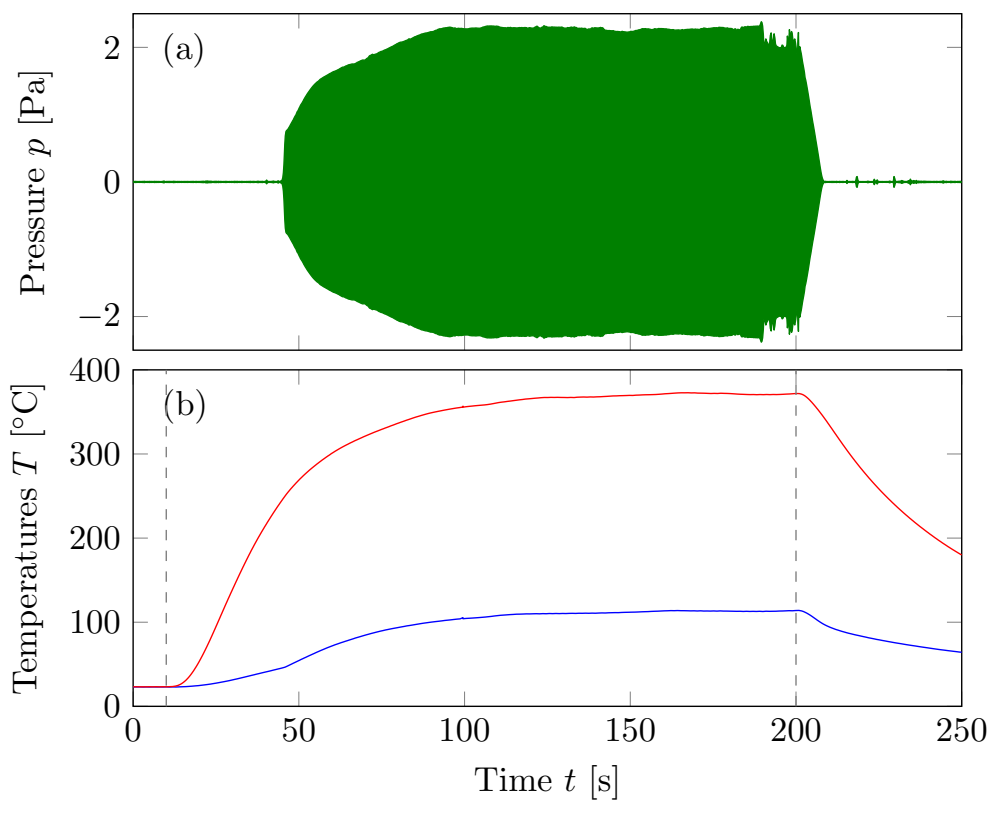

Figure 3. Temporal evolution of (a) the acoustic pressure $p$ at $10 \mathrm{~cm}$ of the opening of the tube and (b) the temperatures $T_{c}$ and $T_{h}$ close to respectively the cold (blue) and hot (red) extremities of the stack. The vertical dashed lines symbolise the instants the flame was lit and blown out.

temperature difference will be minimised is then $r_{h} \sim 250 \mu \mathrm{m}$. Several trials might be required to adjust the quantity of steel wool to obtain the ideal size of pores in the stack.

\subsection{Its performances}

Figure 3(a) shows a recording of the acoustic pressure at $10 \mathrm{~cm}$ of the opening of the tube. The flame is lit at $t=10 \mathrm{~s}$, the engine starts to sing a few tens of seconds later with a maximum sound level around $L_{a c}=98 \mathrm{~dB}_{\mathrm{SPL}}$ (corresponding to peak pressure variations of $2.3 \mathrm{~Pa}$ ). It keeps singing as long as the temperature gradient is maintained by the flame and the soaked paper, in this case until $t=200 \mathrm{~s}$ when the flame is blown out. The temperature distribution in the engine is hard to evaluate. If available, thermocouples can be integrated in the engine to give a coarse insight of the evolution of the temperature, as shown in figure $3(\mathrm{~b})$, even if the incertitude on the position of the thermocouples and their weak coupling with the oscillating fluid do not allow for absolute measurements. The colour of the iron oxide layer at the surface of the steel wool (see insert in figure 2) is however a good indicator of the maximum temperature reached at the hot side of the stack: depending on the flame used, $T_{h}$ goes from $300^{\circ} \mathrm{C}$ to $>400{ }^{\circ} \mathrm{C}$, thus covering a range from purple-blue to gray-blue [18].

Figure 4 shows another view of the same measurements in which a zoom has been performed around the onset of the auto-oscillation, with a focus on the temperature at the cold extremity of the stack. The behaviour of this engine appears simple enough during this period so that different theoretical stages can be qualitatively isolated in accordance with the linear theory of thermoacoustics 8 , 9. At first, the temperature 


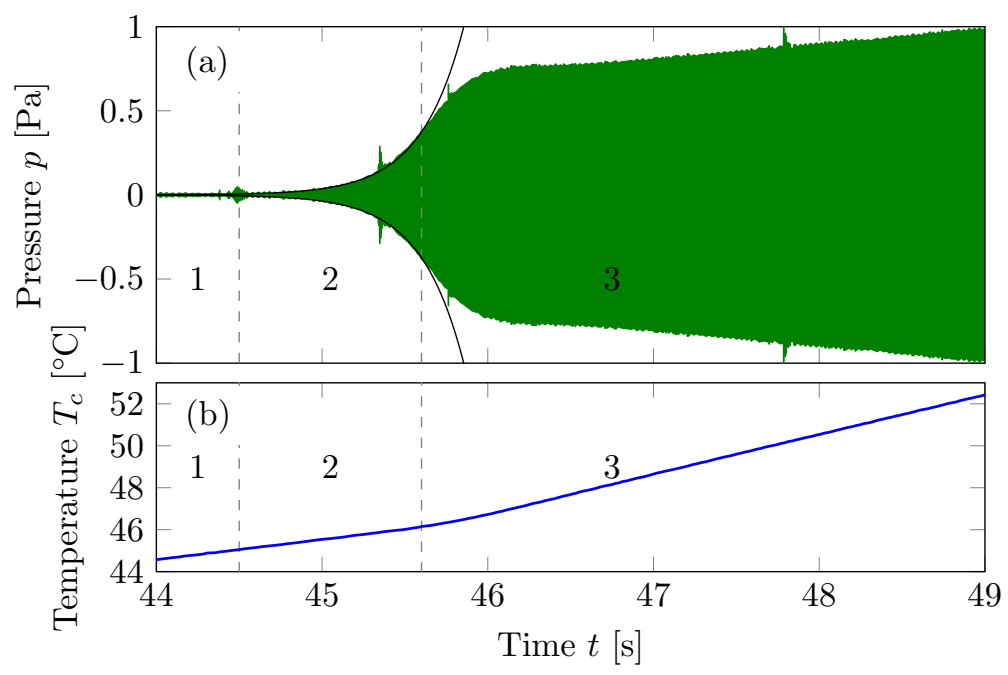

Figure 4. Zoom on the onset of the acoustic instability. Temporal evolution of (a) the acoustic pressure variation at $10 \mathrm{~cm}$ of the opening of the tube and (b) the temperature $T_{c}$ close to the cold extremity of the stack. Three regimes can be distinguished. (1): Stability. (2): Exponential growth of amplitude, fitted with the black curve. (3): Progressive saturation by thermoacoustic heat transport.

gradient $\left(T_{h}-T_{c}\right) / l_{s}$ is not high enough for the thermoacoustic amplification to compensate the losses in the whole set-up. Acoustic perturbations are damped (state labelled 1 , until $t=44.5 \mathrm{~s}$ ). The evolution of the cold temperature $T_{c}$ is at this point controlled mainly by the heat diffusion through the stack. When a sufficient temperature gradient has been reached, any small acoustic perturbation is amplified exponentially at the frequency of the least stable mode of the engine (here the quarterwavelength mode), as is predicted by the linear theory of thermoacoustics [8, 9] (state 2 , from $44.5 \mathrm{~s}$ to $45.6 \mathrm{~s}$ ). The black curves are an exponential fit of the acoustic amplitude. Thermoacoustic amplification then moves away from an exponential growth due to several saturation processes (state 3 ). One of these saturation processes consists of thermoacoustic streaming, namely the advective heat transport from the hot to the cold side of the stack induced by acoustic oscillations. As the amplitude of the acoustic oscillation rises, this acoustic heat pumping adds to the conduction. Thus $T_{c}$ rises at an increased rate and $T_{h}$ grows more slowly: the amplitude growth of the acoustic oscillation slows down and saturates when a thermal equilibrium is reached (i.e. after $\sim 100 \mathrm{~s}$, see figure 3).

The construction of this thermoacoustic engine requires just a few elements, that are common in a scientific laboratory and inexpensive. It can therefore easily be built by students in many copies for further experimental studies, as will be discussed in section 5 .

\section{Kibitsunokama: a rice-based thermoacoustic engine}

An equivalent construction of the traditional Kibitsunokama is proposed here, which itself is inspired from the demonstrator presented by Pr. Ueda during the 2nd International Workshop on Thermoacoustics in Sendai, Japan on May 2014. This 
configuration, in addition to the use of rice as the stack material, has the advantage of requiring a limited temperature difference for its operation: the cold side of the stack remains almost at room temperature, when the hot side only reaches $100^{\circ} \mathrm{C}$.

\subsection{Building it}

Figure 5 (a) shows a photography of different elements that can be used to build a ricebased thermoacoustic engine for less than $30 €$ : long grain rice (1), grids to support the rice (2), a worm gear clamp (3), a fireplace pipe clean-out cap with flat bottom (4), a ventilation duct connector (5), a ventilation duct reducer (6), a flexible foil ventilation ducting (7), or a single wall chimney pipe (8) that can replace elements (5-7) and a $20 \mathrm{cL}$ cup (9). Elements (3) through (8) have a diameter of $125 \mathrm{~mm}$. They can be found in the ventilation or heating area of a local hardware store. The different elements are put together so that the engine total length is $L=46 \mathrm{~cm}$ and the stack is positioned at $h=5.5 \mathrm{~cm}$ of the closed end. One of the grid is rigid enough to support the rice but its perforations are too large and let the rice through. It is stacked with a second grid, finer but pliable, that has been cut out of a frying pan spatter screen. The cup (9) is used to measure both the water quantity and the rice quantity. A schematic view of the assembled system is given on figure 5 (b). The grids supporting the rice are simply jammed in the bulge of the reducer. The position of the stack can easily be modified by adjusting the height of the clamp (3) on the reducer (6). The flexible duct can be extended to adjust the overall length $L$ of the engine. The engine is driven by an electric hotplate, which power is limited to a few hundreds watts. A higher heating power allows to reach the boiling point faster, but the large amount of vapour emitted rapidly warms the stack, thus impeding the thermoacoustic amplification to take place. A video clip, showing the fabrication and a run of two configurations of this engine, has been made available https://youtu.be/dBqa5t8nefo.

The onset condition being defined as the compensation of all losses, working on the tightness of the different seals is primordial to get the longest and loudest sound. Electrical tape between the pieces or duct tape over the seams greatly helps with reducing the acoustical losses.

\subsection{Its operation}

Figure 6 presents measurements of the acoustic pressure variations and the temperature on each side of the layer of rice. The time $t=0$ is the instant at which the engine is placed on the (cold) hotplate, and the power is switched on. Since the engine starts to sing only a few minutes later, the first $10 \mathrm{~min}$ are compressed in the representation. The engine starts to sing when a sufficient temperature difference $T_{h}-T_{c}$ is reached between both sides of the layer of rice, at $t=625 \mathrm{~s}$ in this measurement. The onset of the auto-oscillation takes place after $5 \mathrm{~min}$ to $10 \mathrm{~min}$, depending on the heating power. The working frequency is close to $f \simeq 180 \mathrm{~Hz}$.

The emitted acoustic level is unstable but can exceed $L_{a c} \simeq 116 \mathrm{~dB}_{\mathrm{SPL}}$ (corresponding to $18 \mathrm{~Pa}$ ) at its maximum value measured at $10 \mathrm{~cm}$ from the open end. The sound maintains for $30 \mathrm{~s}$ to several minutes. For the results presented in figure 6 , the singing last for almost 90 seconds, time after which the temperature difference is not enough anymore, due to the homogenisation of the temperature of the rice layer by the steam passing through it. For the same reason, too much heat brings the water to a violent boiling that releases an excessive amount of vapour escaping through the 

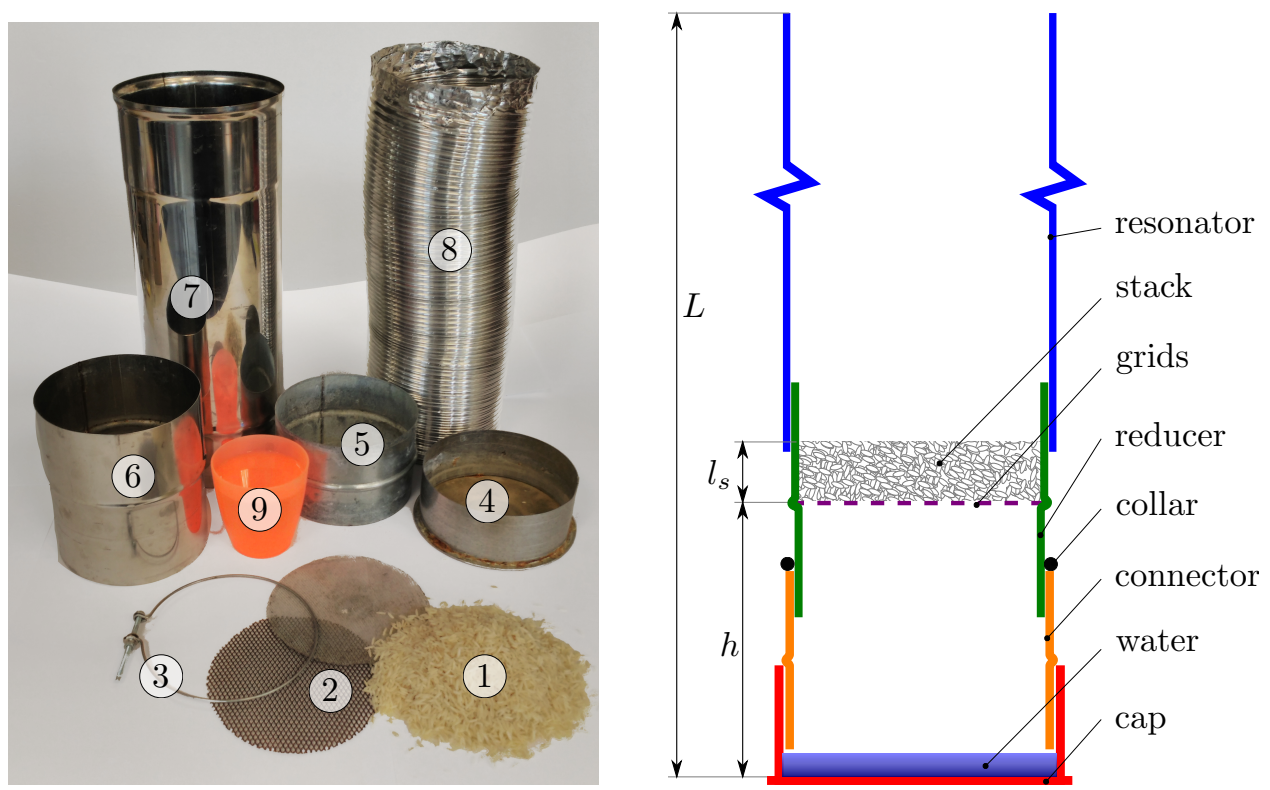

Figure 5. Different elements for the build of a rice-based thermoacoustic engine. (1): long grain rice, (2): grids to support the rice, (3): worm gear clamp, (4): fireplace pipe clean-out cap with flat bottom, (5): ventilation duct connector (6): ventilation duct reducer, (7): flexible foil ventilation ducting, (8): single wall chimney pipe, (9): $20 \mathrm{cL}$ cup. The final assembly is schematically presented on the left.

rice and thus impeding the set-up of the temperature gradient. When the engine stops to sing, taking it off the hotplate sometimes makes it start singing again abruptly, as shown by the measurements on figure 6 at $t=715 \mathrm{~s}$.

Basmati rice is the stack material that offered the best experimental results. Other types of kernels with different size and shapes (cereals or beans) will provide stacks with various porosity and pore size. For example, a green lentils stack works, but the engine sings with a lower acoustic level $L_{a c} \simeq 95 \mathrm{~dB}$ SPL for only a few tens of seconds.

The low temperature operation of this engine is allowed by the presence of water vapour that facilitates the heat exchanges by condensing and evaporating at the interface on the stack during each acoustic cycle. Though the phenomena involved are not yet fully understood, experimental studies [19, 20, have shown that the presence of the vapour can reduce the onset temperature gradient by $\sim 200^{\circ} \mathrm{C}$ for water, and even more for ethanol. The overall behaviour exhibited by this engine is more complex than for the test tube engine. As clearly shown in figure 6 , the device rapidly reaches very high sound levels, but the dynamics of evolution of the amplitude exhibits a complex behaviour with a succession of pulses before the final damping. The reason why such a complicated dynamics is observed is not clear, but it might be related to a competition between different high amplitude effects impacting the temperature distribution in the device. Such kind of dynamics have already been reported in other simple thermoacoustic devices [17, 21] and research works are still in progress to get a deeper understanding of the processes saturating wave amplitude in thermoacoustic oscillators. 


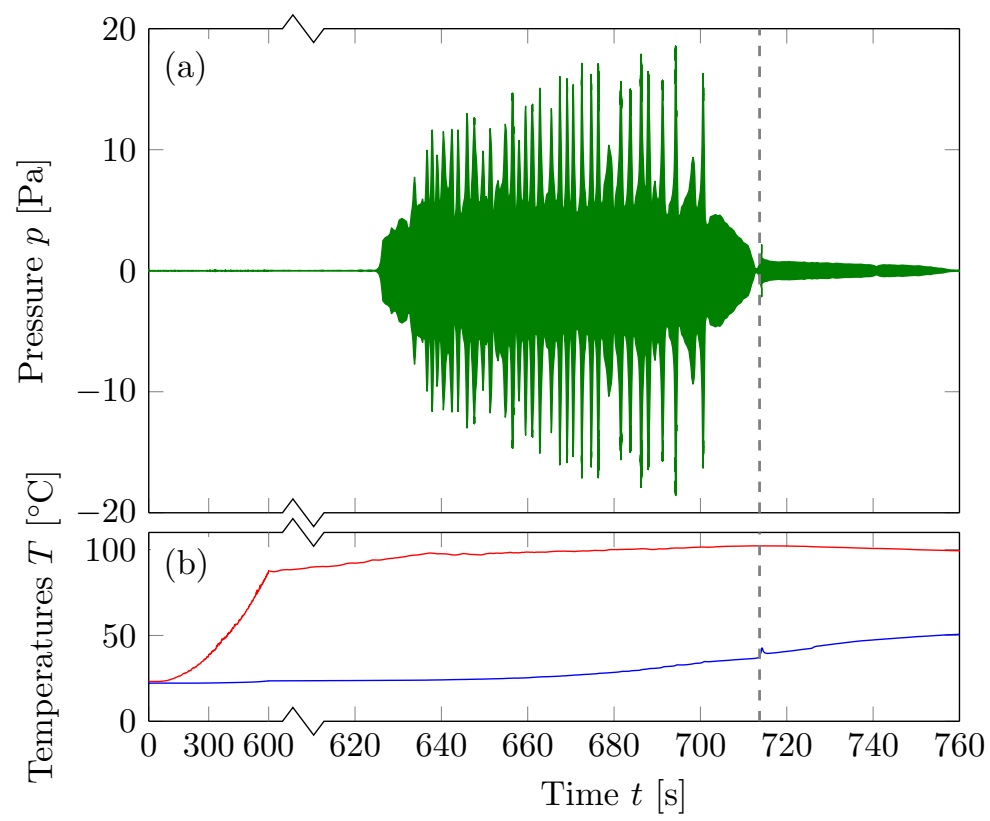

Figure 6. Temporal evolution of (a) the acoustic pressure $p$ at $10 \mathrm{~cm}$ of the opening of the tube and (b) the temperature $T_{c}$ and $T_{h}$ at both extremities of the stack. The vertical gray line shows the moment just after the extinction of the sound, when the engine has been moved from the hotplate to the workbench. After a instant, the short engine starts to sing again. The first ten minutes of heating have been compressed in the representation, the change of slope at $t=600 \mathrm{~s}$ in the hot temperature is due to the change of temporal scale.

\section{Conclusion}

Two demonstrators of thermoacoustic engine have been presented. Based on very simple configurations built from largely available materials, they allow to demonstrate the principle of self-sustained oscillations by thermoacoustic amplification. The construction of such devices is well-suited for under-graduate student's project, and it provides a very demonstrative way to prove that sound can be generated from heat. They are also a basis for further experimental studies.

First of all, parameters which influence the thermoacoustic amplification can be investigated empirically in order to improve the acoustic pressure generated (a basic modelling of the device can even be made using the freely available software DELTAEC [22] developed at Los Alamos National Laboratory). For example, the influences of the stack position along the resonator, of the geometry or material of the stack can be studied. The sound frequency emitted can also be explored by changing the resonator length. The study of the impact of these two parameters can be the starting point to design a thermoacoustic organ pipe [23. Such a musical instrument can be built by using several resonators of different length, each resonator corresponding to one note.

Secondly, these demonstrators can serve to highlight the interest of thermoacoustic engine in an industrial point of view. Hence, the acoustic power generated by the engine can be converted into electric energy by using an alternator (like a loudspeaker 
functioning as a linear alternator 24], a bi-directional turbines [25], or a piezoelectric transducer [26]) or into heat energy by using a thermoacoustic heat-pump [27. The heat energy source provided by the flame can also be replaced by a parabolic solar dish concentrating the sun's rays on the hot side of the stack.

Finally, more academic experiments for pedagogical demonstrations can also be imagined to explore the use of a thermoacoustic oscillator as a specific kind of autonomous, dynamical system. For instance, the coupling of several oscillators 28, 29, or the forcing of a thermoacoustic oscillator by an external sound source [30] leads to synchronisation phenomena and other nonlinear processes (quenching, chaos [31). Therefore, thermoacoustic oscillators could be used advantageously for pedagogical purposes in a class devoted to dynamical systems.

\section{References}

[1] AD Pierce. Acoustics: an introduction to its physical principles and applications. McGraw-Hill, New York, 1981.

[2] S Temkin. Elements of acoustics. Wiley, New York, 1981.

[3] JW Strutt (Baron Rayleigh). The theory of sound. Macmillan and Co., London, 1877.

[4] C Sondhauss. Über die Schallschwingungen der Luft in erhitzten Glasröhren und in gedeckten Pfeifen von ungleicher Weite. Ann. Phys. Berlin, 155(1):1-34, 1850. http://dx.doi.org/ 10.1002/andp. 18501550102

[5] PL Rijke. Notiz über eine neue Art, die in einer an beiden Enden offenen Röhre enthaltene Luft in Schwingungen zu versetzen. Ann. Phys. Berlin, 183(6):339-343, 1859. http: //dx.doi.org/10.1002/andp.18591830616

[6] TC Lieuwen. Unsteady combustor physics. Cambridge University Press, 2012. ISBN: 978-1107-01599-9.

[7] A Ueda. Ugetsu Monogatari (Tales of Moonlight and Rain). 1776 (orig. Jap. publ.), 1938-1941 (1st Eng. trad. by W Whitehouse as Tales of a Clouded Moon). Translation available in most languages.

[8] N Rott. Thermoacoustics. In Adv. Appl. Mech., volume 20 of Adv. Appl. Mech., pp.135-175. Elsevier, 1980. http://dx.doi.org/10.1016/S0065-2156(08)70233-3

[9] GW Swift. Thermoacoustic engines. J. Acoust. Soc. Am., 84(4):1145-1180, 1988. http: //dx.doi.org/10.1121/1.396617.

[10] SL Garrett. Resource letter: Ta-1: thermoacoustic engines and refrigerators. Am. J. Phys., 72(1):11 - 17, 2004. http://dx.doi.org/10.1119/1.1621034.

[11] SL Garrett and S Backhaus. The power of sound. Am. Sci., 88(6):516-525, 2000. http: //dx.doi.org/10.1511/2000.6.516

[12] SL Garrett. "Acoustic Laser" Kit Instructions. http://www.acs.psu.edu/thermoacoustics/ refrigeration/Demo_Inst5(no_specials).pdf (Kit no more available for purchase).

[13] A Jeromen. A simplified thermoacoustic engine demonstration. Am. J. Phys., 71(5):496-499, 2003. http://dx.doi.org/10.1119/1.1524163

[14] KJ Bastyr and RM Keolian. High-frequency thermoacoustic-Stirling heat engine demonstration device. Acoust. Res. Lett. Online, 4(2):37-40, 2003. http://dx.doi.org/10.1121/1.1558931

[15] KI Matveev, N Shafiei-Tehrany, and CD Richards. Small-scale thermoacoustic engine demonstrator. In Proc. of 8th Int. Workshop on micro and nanotechnology for power generation and energy conversion applications, Sendai (Jp), 2008.

[16] DA Russell and P Weibull. Tabletop thermoacoustic refrigerator for demonstrations. Am. J. Phys., 70(12):1231-1233, 2002. http://dx.doi.org/10.1119/1.1485720

[17] M Guedra, G Penelet, and P Lotton. Experimental and theoretical study of the dynamics of self-sustained oscillations in a standing wave thermoacoustic engine. J. Appl. Phys., 115(2):024504, 2014. http://dx.doi.org/10.1063/1.4861879

[18] Tempering (metallurgy). In Wikipedia. https://en.wikipedia.org/wiki/Tempering_ (metallurgy)\#Tempering_colors. Accessed: 2016-05-17.

[19] D Noda and Y Ueda. A thermoacoustic oscillator powered by vaporized water and ethanol. Am. J. Phys., 81(2):124-126, 2013. http://dx.doi.org/10.1119/1.4766940

[20] K Tsuda and Y Ueda. Abrupt reduction of the critical temperature difference of a thermoacoustic engine by adding water. In Proc. of 20th Int. Symp. on Nonlinear Acoustics, Vol.5, p.097173, 2015. http://dx.doi.org/10.1063/1.4932036 
Do It Yourself: make your own thermoacoustic engine with steel wool or rice.

[21] Z Yu, AJ Jaworski, and AS Abduljalil. Fishbone-like instability in a looped-tube thermoacoustic engine. J. Acoust. Soc. Am., 128(4):EL188-EL194, 2010. http://dx.doi.org/10.1121/1. 3486198

[22] WC Ward, J Clark, and GW Swift. Design Environment for Low-amplitude Thermoacoustic Energy Conversion (DeltaEC Version 6.2) Users Guide, 2008.

[23] N Stodola, B Tillotson, and B Bruno. Development of a thermoacoustic organ. J. Acoust. Soc. Am., 118(3):1926-1927, 2005. http://dx.doi.org/10.1121/1.4780642

[24] Z Yu, P Saechan, and AJ Jaworski. A method of characterising performance of audio loudspeakers for linear alternator applications in low-cost thermoacoustic electricity generators. Appl. Acoust., 72(5):260-267, 2011. http://dx.doi.org/10.1016/j.apacoust. 2010.11.011

[25] K deBlok, P Owczarek, and M-X Franois. Bi-directional turbines for converting acoustic wave power into electricity. In Proc. of 9th PAMIR Int. Conf. on Fundamental and Applied MHD, Riga (Lv), 2014.

[26] C Jensen and R Raspet. Thermoacoustic power conversion using a piezoelectric transducer. J. Acoust. Soc. Am., 128(1):98-103, 2010. http://dx.doi.org/10.1121/1.3409370

[27] DL Gardner and CQ Howard. Waste-heat-driven thermoacoustic engine and refrigerator. In Proc. of Acoustics, Adelaide (Au), 2009.

[28] PS Spoor and GW Swift. The Huygens entrainment phenomenon and thermoacoustic engines. J. Acoust. Soc. Am., 108(2):588-599, 2000. http://dx.doi.org/10.1121/1.429590.

[29] T Biwa, S Tozuka, and T Yazaki. Amplitude death in coupled thermoacoustic oscillators. Phys. Rev. Appl., 3(3):034006, 2015. http://dx.doi.org/10.1103/PhysRevApplied.3.034006

[30] G Penelet and T Biwa. Synchronization of a thermoacoustic oscillator by an external sound source. Am. J. Phys., 81(4):290, 2013. http://dx.doi.org/10.1119/1.4776189

[31] T Yazaki. Experimental observation of thermoacoustic turbulence and universal properties at the quasiperiodic transition to chaos. Phys. Rev. E, 48(3):1806, 1993. http://dx.doi.org/ 10.1103/PhysRevE.48.1806 